\title{
Article \\ Geometric Constants in Banach Spaces Related to the Inscribed Quadrilateral of Unit Balls
}

\author{
Asif Ahmad (D), Qi Liu *(D) and Yongjin Li (D) \\ School of Mathematics, Sun Yat-sen University, Guangzhou 510275, China; ahmad7@mail2.sysu.edu.cn (A.A.); \\ stslyj@mail.sysu.edu.cn (Y.L.) \\ * Correspondence: liuq325@mail2.sysu.edu.cn
}

Citation: Ahmad, A.; Liu, Q.; Li, Y. Geometric Constants in Banach Spaces Related to the Inscribed Quadrilateral of Unit Balls. Symmetry 2021, 13, 1294. https://doi.org/ $10.3390 /$ sym 13071294

Academic Editor: Roman Ger

Received: 4 July 2021

Accepted: 18 July 2021

Published: 19 July 2021

Publisher's Note: MDPI stays neutral with regard to jurisdictional claims in published maps and institutional affiliations.

Copyright: (c) 2021 by the authors. Licensee MDPI, Basel, Switzerland. This article is an open access article distributed under the terms and conditions of the Creative Commons Attribution (CC BY) license (https:// creativecommons.org/licenses/by/ $4.0 /)$.
Abstract: We introduce a new geometric constant $\operatorname{Jin}(X)$ based on a generalization of the parallelogram law, which is symmetric and related to the length of the inscribed quadrilateral side of the unit ball. We first investigate some basic properties of this new coefficient. Next, it is shown that, for a Banach space, $\operatorname{Jin}(X)$ becomes 16 if and only if the norm is induced by an inner product. Moreover, its properties and some relations between other well-known geometric constants are studied. Finally, a sufficient condition which implies normal structure is presented.

Keywords: Banach spaces; geometric constants; geometry of normed spaces; normal structure

\section{Introduction}

In recent years, many geometric constants have been defined and studied in the literature, which makes it easier for us to deal with some problems in Banach space, because it can describe the geometric properties of space quantitatively. These geometric constants have mathematical beauty, and there are countless relationships between different geometric constants. One of the best known is the von Neumann-Jordan constant $C_{\mathrm{NJ}}(X)$ and the James constant $J(X)$. Readers interested in this field are advised to see [1-6] and the references mentioned therein. It is worth mentioning that geometric constants play a vital role as a tool for solving other problems, such as in the study of Banach-Stone theorem, Bishop-Phelps-Bollobás theorem, and Tingley's Problem. These are important research topics in the area of functional analysis and we recommend that readers refer to the literature [7-9].

Among all normed spaces, the Hilbert spaces are generally considered to have the simplest and clearest geometric structure. Many mathematicians have found the conditions on normed spaces under which such spaces become inner product spaces. Results of this kind are of importance in functional analysis-for example, in the theory of operator algebras and certain stability problems $[10,11]$. In addition, since every physical system is associated with a Hilbert space, the notion of inner product space also plays a crucial role in quantum mechanics; see [12]. The rich theory of Hilbert spaces has been created by the efforts of many mathematicians; refer to [13-15] for more details. It is worth noting that the background meaning of many famous geometric constants is closely related to the description of inner product spaces.

The first norm characterization of inner product spaces was given by Fréchet [16] in 1935.

Lemma 1 ([16]). A complex normed space $(X,\|\cdot\|)$ is an inner product space if and only if

$$
\left\|x_{1}+x_{2}\right\|^{2}+\left\|x_{2}+x_{3}\right\|^{2}+\left\|x_{1}+x_{3}\right\|^{2}=\left\|x_{1}+x_{2}+x_{3}\right\|^{2}+\left\|x_{1}\right\|^{2}+\left\|x_{2}\right\|^{2}+\left\|x_{3}\right\|^{2}
$$

for all $x_{1}, x_{2}, x_{3} \in X$. 
If we consider the usual Euclidean space $\left(R^{n},\|\cdot\|\right)$, then the identity $\|x+y\|^{2}+\| x-$ $y\left\|^{2}=2\right\| x\left\|^{2}+2\right\| y \|^{2}$ is called the parallelogram law, and it is well known. This identity can be naturally extended to the more general case. Research on the equivalent characterization of inner product space has been attracting much attention. We refer the readers to $[17,18]$ for more details.

M. M. Day [19] gave the following weaker characterization, referred to as "the rhombus law".

Lemma $2([19])$. Let $(X,\|\cdot\|)$ be a real normed linear space. Then, $\|\cdot\|$ derives from an inner product if and only if

$$
\left\|x_{1}+x_{2}\right\|^{2}+\left\|x_{1}-x_{2}\right\|^{2} \sim 4
$$

for all $x_{1}, x_{2} \in S_{X}$, where $\sim$ stands either for $\leq$ or for $\geq$.

\section{Preliminaries}

We now give some definitions related to geometric constants. Let $X$ be a real Banach space with $\operatorname{dim} X \geq 2$ and denote by $S_{X}$ and $B_{X}$ the unit sphere and the unit ball, respectively.

In combination with Jordan and von Neumann's [20] brilliant work on the characterization of inner product spaces by the parallelogram law, Clarkson [21] first proposed the von Neumann-Jordan constant $C_{\mathrm{NJ}}(X)$ of Banach space. More precisely, the von Neumann-Jordan constant of $X$ is defined by

$$
C_{\mathrm{NJ}}(X)=\sup \left\{\frac{\left\|x_{1}+x_{2}\right\|^{2}+\left\|x_{1}-x_{2}\right\|^{2}}{2\left(\left\|x_{1}\right\|^{2}+\left\|x_{2}\right\|^{2}\right)}: x_{1}, x_{2} \in X,\left(x_{1}, x_{2}\right) \neq(0,0)\right\} \text {. }
$$

The James constant $J(X)$ of a Banach space $X$ is introduced by Gao and Lau [22] as follows:

$$
J(X)=\sup \left\{\min \left\{\left\|x_{1}+x_{2}\right\|,\left\|x_{1}-x_{2}\right\|\right\}: x_{1}, x_{2} \in S_{X}\right\} .
$$

Moreover, the various properties of these constants are given in [22-24]:

(1) $\sqrt{2} \leq J(X) \leq 2$.

(2) $J(X)=\sqrt{2}$ whenever $X$ represents Hilbert space; the converse is not correct.

(3) $1 \leq C_{\mathrm{NJ}}(X) \leq 2$.

(4) $X$ is a Hilbert space if $C_{\mathrm{NJ}}(X)=1$.

(5) $X$ is uniformly non-square if $C_{\mathrm{NJ}}(X)<2$.

(6) $C_{\mathrm{NJ}}(X)=C_{\mathrm{NJ}}\left(X^{*}\right)$.

The modified von Neumann-Jordan constant

$$
C_{\mathrm{NJ}}^{\prime}(X):=\sup \left\{\frac{\left\|x_{1}+x_{2}\right\|^{2}+\left\|x_{1}-x_{2}\right\|^{2}}{4}: x_{1}, x_{2} \in S_{X}\right\}
$$

was studied by Takahashi [25] and Alonso et al. [2].

Recall that the Banach space $X$ is called uniformly non-square [26] if there exists a $\delta \in(0,1)$ such that for any $x_{1}, x_{2} \in S_{X}$ either $\frac{\left\|x_{1}+x_{2}\right\|}{2} \leq 1-\delta$ or $\frac{\left\|x_{1}-x_{2}\right\|}{2} \leq 1-\delta$. It is known that $X$ is uniformly non-square if and only if $C_{\mathrm{NJ}}^{\prime}(X)<2$.

Definition 1 ([27]). A Banach space $(X,\|\cdot\|)$ is non-square if, for every $x_{1}, x_{2} \in S_{X}$, we have

$$
\min \left\{\left\|x_{1}+x_{2}\right\|,\left\|x_{1}-x_{2}\right\|\right\}<2
$$

Baronti et al. defined the following two new constants, which are related to the perimeter of an inscribed triangle in a semicircle of normed space. 
Definition 2 ([28]).

$$
A_{1}(X)=\frac{1}{2} \inf _{x_{1} \in S_{X}} \sup _{x_{2} \in S_{X}}\left(\left\|x_{1}-x_{2}\right\|+\left\|x_{1}+x_{2}\right\|\right)
$$

and

$$
A_{2}(X)=\frac{1}{2} \sup _{x_{1} \in S_{X}} \sup _{x_{2} \in S_{X}}\left(\left\|x_{1}-x_{2}\right\|+\left\|x_{1}+x_{2}\right\|\right)
$$

Javier Alonso and Enrique Llorens-Fuster [29] also introduced the constants

$$
\begin{gathered}
t(x)=\inf _{x_{1} \in S_{X}} \sup _{x_{2} \in S_{X}} \sqrt{\left\|x_{1}+x_{2}\right\|\left\|x_{1}-x_{2}\right\|}, \\
T(x)=\sup _{x_{1}, x_{2} \in S_{X}} \sqrt{\left\|x_{1}+x_{2}\right\|\left\|x_{1}-x_{2}\right\| .}
\end{gathered}
$$

It is intuitive to show that the geometric meaning of $t(x)$ and $T(x)$ is the geometric mean of the diagonals of a "rhombus".

Fu et al. also introduced the constants:

$$
\begin{gathered}
J_{L}(X)=\inf \left\{\left\|x_{1}-x_{2}\right\|,\left\|x_{2}-x_{3}\right\|,\left\|x_{1}-x_{3}\right\|:\left\|x_{1}\right\|=\left\|x_{2}\right\|=\left\|x_{3}\right\|=1, x_{1}+x_{2}+x_{3}=0\right\} ; \\
Y_{J}(X)=\sup \left\{\left\|x_{1}-x_{2}\right\|,\left\|x_{2}-x_{3}\right\|,\left\|x_{1}-x_{3}\right\|:\left\|x_{1}\right\|=\left\|x_{2}\right\|=\left\|x_{3}\right\|=1, x_{1}+x_{2}+x_{3}=0\right\},
\end{gathered}
$$

which are related to the side lengths of the inscribed triangles of unit balls to study the geometric properties of Banach spaces. Moreover, the various properties of these constants are given in [30]:

(1) $1 \leq J_{L}(X) \leq 2, \sqrt{3} \leq Y_{J}(X) \leq 2$.

(2) Let $X$ be a Hilbert space, then $J_{L}(X)=Y_{J}(X)=\sqrt{3}$.

(3) $\frac{2}{J(X)} \leq J_{L}(X) \leq Y_{J}(X) \leq \sqrt{4 J(X)-1}$.

Inspired by the above constants, and in combination with the characterization of the inner product space, we define a new geometric constant $\operatorname{Jin}(X)$ and some related properties are discussed. Furthermore, we attempt to relate this to other important geometric concepts and finally give an application that is closely related to normal structure.

The paper is organized as follows: we exhibit some basic properties of this new coefficient in the next section. Furthermore, the connections between Hilbert spaces are investigated. The relationship between the constant $\operatorname{Jin}(X)$ and other well-known constants is emphasized in terms of nontrivial inequalities. In Section 4, we establish a new necessary condition for Banach spaces with normal structure in the form of $\operatorname{Jin}(X)$.

\section{Constant $\operatorname{Jin}(X)$}

It is well known that the circle and its inscribed polygon is an important research topic in Euclidean geometry. The results of this study reveal many important geometric properties of Euclidean planes. Some results have been generalized to Banach spaces, such as orthogonality, angle, circumference, and other geometric concepts. Instead of Euclidean plane geometry, we consider a more general case, in the framework of a Banach space. We can invesigate the Banach space by considering its unit sphere, because the unit sphere can largely reflect the geometric properties of a space $X$, such as the well-known convexity. According to the result of [31], in many cases, we only need to consider the extreme points on the unit ball to study the geometric constant; for some classical Banach spaces, such as $l_{1}, l_{\infty}$, the two dimensional case, the number of its extreme points on the unit ball is four or more. In a way, our four variables are significant, either in terms of the four sides of an inscribed quadrilateral and its two diagonals to understand, or through four variables, which are special points. Considering these factors, we begin by introducing the following key definition: 


\section{Definition 3.}

$$
\begin{aligned}
\operatorname{Jin}(X) & =\sup \left\{\sum\left\|x_{i}-x_{j}\right\|^{2}: x_{i}, x_{j} \in S_{X}, 1 \leq i<j \leq 4, i, j \in\{1,2,3,4\}, \sum_{i=1}^{4} x_{i}=0\right\} \\
& =\sup \left\{\left\|x_{1}-x_{2}\right\|^{2}+\left\|x_{2}-x_{3}\right\|^{2}+\left\|x_{1}-x_{3}\right\|^{2}+\left\|x_{1}-x_{4}\right\|^{2}\right. \\
& \left.+\left\|x_{2}-x_{4}\right\|^{2}+\left\|x_{3}-x_{4}\right\|^{2}: x_{1}, x_{2}, x_{3}, x_{4} \in S_{X}, x_{1}+x_{2}+x_{3}+x_{4}=0\right\} .
\end{aligned}
$$

In fact, upon observation, we find that the constant $\operatorname{Jin}(X)$ is symmetric because the positions of $x_{1}, x_{2}, x_{3}$, and $x_{4}$ are interchangeable, and point $x_{1}+x_{2}$ is actually symmetric to point $x_{3}+x_{4}$. The constant $\operatorname{Jin}(X)$ can be understood in a very intuitive way: the sum of the squares of the lengths of the four sides of the inscribed quadrilateral plus the sum of the squares of the two diagonals.

Proposition 1. Suppose that $X$ is a normed space. Then,

$$
\operatorname{Jin}(X) \geq 8 C_{\mathrm{NJ}}^{\prime}(X)+8 .
$$

Proof. Assume $x_{1}=x, x_{2}=-x, x_{3}=y, x_{4}=-y$ and $x, y \in S_{X}$; then, we have

$$
\begin{aligned}
& \left\|x_{1}-x_{2}\right\|^{2}+\left\|x_{2}-x_{3}\right\|^{2}+\left\|x_{1}-x_{3}\right\|^{2} \\
& +\left\|x_{1}-x_{4}\right\|^{2}+\left\|x_{2}-x_{4}\right\|^{2}+\left\|x_{3}-x_{4}\right\|^{2} \\
& =\|2 x\|^{2}+\|x+y\|^{2}+\|x-y\|^{2} \\
& +\|x+y\|^{2}+\|x-y\|^{2}+\|2 y\|^{2} \\
& =2\|x+y\|^{2}+2\|x-y\|^{2}+8 .
\end{aligned}
$$

Hence, we can deduce that

$$
\operatorname{Jin}(X) \geq 8 C_{\mathrm{NJ}}^{\prime}(X)+8 .
$$

From Proposition 1, we can obtain the following estimate.

Proposition 2. Suppose that $X$ is a normed space. Then,

$$
16 \leq \operatorname{Jin}(X) \leq 24
$$

Proof. Using the same method as in Proposition 1, we can conclude that

$$
\operatorname{Jin}(X) \geq 2\|x+y\|^{2}+2\|x-y\|^{2}+8
$$

for any $x, y \in S_{X}$, letting $x=-y$ and hence $\operatorname{Jin}(X) \geq 16$.

The latter assertion can be derived from the following estimate

$$
\left\|x_{1}+x_{2}\right\|^{2} \leq 2\left\|x_{1}\right\|^{2}+2\left\|x_{2}\right\|^{2}
$$

as desired.

Next, we give the following two examples, which illustrate the relationship between the constant $\operatorname{Jin}(X)$ and the four variables.

Example 1. Let $X$ be $\mathbb{R}^{2}$ with the norm defined by

$$
\left\|\left(x_{1}, x_{2}\right)\right\|=\max \left\{\left|x_{1}\right|,\left|x_{2}\right|\right\} .
$$


Letting $x_{1}=(1,1), x_{2}=(1,-1), x_{3}=(-1,1), x_{4}=(-1,-1)$, it is easy to see that

$$
\left\|x_{1}-x_{2}\right\|=\left\|x_{2}-x_{3}\right\|=\left\|x_{1}-x_{3}\right\|=\left\|x_{1}-x_{4}\right\|=\left\|x_{2}-x_{4}\right\|=\left\|x_{3}-x_{4}\right\|=2
$$

and $x_{1}+x_{2}+x_{3}+x_{4}=0$. Hence, we can obtain $\operatorname{Jin}(X)=24$.

Example 2. Let $X$ be $\mathbb{R}^{2}$ with the norm defined by

$$
\left\|\left(x_{1}, x_{2}\right)\right\|=\left|x_{1}\right|+\left|x_{2}\right| .
$$

Letting $x_{1}=(1,0), x_{2}=(-1,0), x_{3}=(0,1), x_{4}=(0,-1)$, we then have

$$
\left\|x_{1}-x_{2}\right\|=\left\|x_{2}-x_{3}\right\|=\left\|x_{1}-x_{3}\right\|=\left\|x_{1}-x_{4}\right\|=\left\|x_{2}-x_{4}\right\|=\left\|x_{3}-x_{4}\right\|=2
$$

and $x_{1}+x_{2}+x_{3}+x_{4}=0$. Thus, $\operatorname{Jin}(X)=24$.

Proposition 3. Suppose that $X$ is a normed space. Then,

$$
\operatorname{Jin}(X) \geq 4 J(X)^{2}+8 .
$$

Proof. Using the same method as in Proposition 1 and applying the elementary inequality, we can conclude that

$$
\begin{aligned}
\operatorname{Jin}(X) & \geq 2\left\|x_{1}+x_{2}\right\|^{2}+2\left\|x_{1}-x_{2}\right\|^{2}+8 \\
& =4 \frac{\left\|x_{1}+x_{2}\right\|^{2}+\left\|x_{1}-x_{2}\right\|^{2}}{2}+8 \\
& \geq 4 \min \left\{\left\|x_{1}+x_{2}\right\|^{2},\left\|x_{1}-x_{2}\right\|^{2}\right\}+8
\end{aligned}
$$

for any $x_{1}, x_{2} \in S_{X}$. Hence,

$$
\operatorname{Jin}(X) \geq 4 J(X)^{2}+8
$$

Theorem 1. Let $X$ be a Banach space. Then, $\operatorname{Jin}(X)=16$ if and only if $X$ is a Hilbert space.

Proof. By Lemma 1, we can deduce that

$$
\left\|x_{1}+x_{2}\right\|^{2}+\left\|x_{2}+x_{3}\right\|^{2}+\left\|x_{1}+x_{3}\right\|^{2}=4
$$

for any $x_{1}, x_{2}, x_{3}, x_{4} \in S_{X}, x_{1}+x_{2}+x_{3}+x_{4}=0$.

Applying the parallelogram law, we obtain that

$$
\begin{aligned}
& \left\|x_{1}+x_{2}\right\|^{2}+\left\|x_{1}-x_{2}\right\|^{2} \\
& +\left\|x_{2}+x_{3}\right\|^{2}+\left\|x_{2}-x_{3}\right\|^{2} \\
& +\left\|x_{1}+x_{3}\right\|^{2}+\left\|x_{1}-x_{3}\right\|^{2} \\
& =12 .
\end{aligned}
$$

Thus, we have

$$
\left\|x_{1}-x_{2}\right\|^{2}+\left\|x_{2}-x_{3}\right\|^{2}+\left\|x_{1}-x_{3}\right\|^{2}=8 .
$$

Analogously, we can prove that

$$
\begin{aligned}
& \left\|x_{1}-x_{2}\right\|^{2}+\left\|x_{2}-x_{4}\right\|^{2}+\left\|x_{1}-x_{4}\right\|^{2}=8 \\
& \left\|x_{2}-x_{3}\right\|^{2}+\left\|x_{2}-x_{4}\right\|^{2}+\left\|x_{3}-x_{4}\right\|^{2}=8
\end{aligned}
$$




$$
\left\|x_{1}-x_{3}\right\|^{2}+\left\|x_{1}-x_{4}\right\|^{2}+\left\|x_{3}-x_{4}\right\|^{2}=8 .
$$

From the above, it follows that

$$
\left\|x_{1}-x_{2}\right\|^{2}+\left\|x_{2}-x_{3}\right\|^{2}+\left\|x_{1}-x_{3}\right\|^{2}+\left\|x_{1}-x_{4}\right\|^{2}+\left\|x_{2}-x_{4}\right\|^{2}+\left\|x_{3}-x_{4}\right\|^{2}=16 .
$$

For the second part of the proof, we suppose $\operatorname{Jin}(X)=16$. Then, we have

$$
\left\|x_{1}-x_{2}\right\|^{2}+\left\|x_{2}-x_{3}\right\|^{2}+\left\|x_{1}-x_{3}\right\|^{2}+\left\|x_{1}-x_{4}\right\|^{2}+\left\|x_{2}-x_{4}\right\|^{2}+\left\|x_{3}-x_{4}\right\|^{2} \leq 16
$$

for any $x_{1}, x_{2}, x_{3}, x_{4} \in S_{X}, x_{1}+x_{2}+x_{3}+x_{4}=0$.

Letting $x_{1}=x, x_{2}=-x, x_{3}=y, x_{4}=-y$ and $x, y \in S_{X}$, we obtain

$$
\begin{aligned}
& \left\|x_{1}-x_{2}\right\|^{2}+\left\|x_{2}-x_{3}\right\|^{2}+\left\|x_{1}-x_{3}\right\|^{2} \\
& \quad+\left\|x_{1}-x_{4}\right\|^{2}+\left\|x_{2}-x_{4}\right\|^{2}+\left\|x_{3}-x_{4}\right\|^{2} \\
& =2\|x+y\|^{2}+2\|x-y\|^{2}+\|2 y\|^{2}+\|2 y\|^{2} \\
& \leq 16 .
\end{aligned}
$$

Consequently,

$$
\|x+y\|^{2}+\|x-y\|^{2} \leq 4
$$

for any $x, y \in S_{X}$. Thus, $X$ is a Hilbert space directly from Lemma 2.

Proposition 4. Let $X$ be a Banach space; if $X$ is not non-square, then $\operatorname{Jin}(X)=24$.

Proof. First note that $X$ is not non-square and therefore there exist $x_{n}, y_{n} \in S_{X}$ for which

$$
\left\|x_{n}+y_{n}\right\| \rightarrow 2,\left\|x_{n}-y_{n}\right\| \rightarrow 2(n \rightarrow \infty) .
$$

Letting $x_{1}=x_{n}, x_{2}=-x_{n}, x_{3}=y_{n}, x_{4}=-y_{n}$ and $x_{n}, y_{n} \in S_{X}$, we obtain

$$
\begin{aligned}
& \left\|x_{1}-x_{2}\right\|^{2}+\left\|x_{2}-x_{3}\right\|^{2}+\left\|x_{1}-x_{3}\right\|^{2} \\
& \quad+\left\|x_{1}-x_{4}\right\|^{2}+\left\|x_{2}-x_{4}\right\|^{2}+\left\|x_{3}-x_{4}\right\|^{2} \\
& =2\left\|x_{n}+y_{n}\right\|^{2}+2\left\|x_{n}-y_{n}\right\|^{2}+8,
\end{aligned}
$$

which implies that $\operatorname{Jin}(X)=24$.

Proposition 4 is thereby proven.

Contractive mappings are closely related to the fixed point property, which plays an important role in the application of Banach space geometry. It is worth noting that contractive mappings have attracted more interest in other scientific branches; refer to [32-34] for more details. Garca-Falset et al. [35] proved that uniformly non-square Banach space has a fixed point property. Next, we establish a necessary condition for Banach spaces that have a fixed point property in the form of $\operatorname{Jin}(X)$.

Proposition 5. Assume $X$ is a Banach space with $\operatorname{Jin}(X)<24$; then, $X$ has the fixed point property.

Proof. By Proposition 1, we obtain that

$$
C_{\mathrm{NJ}}^{\prime}(X) \leq \frac{\operatorname{Jin}(X)-8}{8}
$$

This implies that $C_{\mathrm{NJ}}^{\prime}(X)<2$ and hence $X$ is uniformly non-square. Thus, we can deduce that $X$ has the fixed point property (see [35]), as desired. 


\section{The Coefficient of Weak Orthogonality}

Definition 4 ([36]). A nonempty bounded and convex subset $K$ of a Banach space $X$ is said to have normal structure if, for every convex subset $H$ of $K$ that contains more than one point, there exists a point $x_{0} \in H$ such that

$$
\sup \left\{\left\|x_{0}-x_{1}\right\|: x_{1} \in H\right\}<\sup \left\{\left\|x_{1}-x_{2}\right\|: x_{1}, x_{2} \in H\right\} .
$$

A Banach space $X$ is said to have weak normal structure if each weakly compact convex set $K$ in $X$ that contains more than one point has normal structure. Obviously, for a reflexive Banach space, weak normal structure and normal structure coincide. Let $Y$ be a subset of a Banach space $X$. A mapping $g: Y \rightarrow Y$ is called a nonexpansive mapping if $\left\|g x_{1}-g x_{2}\right\| \leq\left\|x_{1}-x_{2}\right\|$ for any $x_{1}, x_{2} \in Y$. A large body of literature shows that normal structure plays an important role in the fixed point theory of nonexpansive mappings (see [37]).

Definition 5 ([38]). A Banach space X is said to have the property WORTH whenever

$$
\limsup _{n \rightarrow \infty}\left|\left\|z_{n}+z\right\|-\left\|z_{n}-z\right\|\right|=0
$$

for all weakly null sequences $z_{n}$ in $X$ and all the elements $z$ of $X$.

Years later, Sims introduced a parameter [39] on the original basis:

$$
w(X)=\sup \left\{\lambda>0: \lambda \cdot \liminf _{n \rightarrow \infty}\left\|z_{n}+z\right\| \leq \liminf _{n \rightarrow \infty}\left\|z_{n}-z\right\|\right\}
$$

where the supremum takes over all the weakly null sequences $z_{n}$ in $X$ and all the elements $z$ of $X$. It was proven that $\frac{1}{3} \leq w(X) \leq 1$ for all Banach space $X$.

We begin by starting with two lemmas, which will be our main tools.

Lemma 3 ([40]). Let X be a Banach space without weak normal structure; then, for any $0<\varepsilon<1$, there exists a sequence $\left\{z_{n}\right\} \subseteq S_{X}$ with $z_{n} \stackrel{w}{\longrightarrow} 0$, and

$$
1-\varepsilon<\left\|z_{n+1}-z\right\|<1+\varepsilon
$$

for sufficiently large $n$, and any $z \in \operatorname{co}\left\{z_{k}\right\}_{k=1}^{n}$.

From Lemma 3, we obtain the following:

Lemma 4. Let $X$ be a Banach space without weak normal structure; then, for any $0<\varepsilon<w(X)$, there exists an $z_{n}$ in $S_{X}$, satisfying

(i) $1-\varepsilon \leq\left\|z_{n}-z\right\| \leq 1+\varepsilon$;

(ii) $\left\|z_{n}-z_{1}\right\| \leq 1+\varepsilon$;

(iii) $\left\|z_{n}+x_{1}\right\| \leq \frac{1+\varepsilon}{w(X)-\varepsilon}$,

where $z \in \operatorname{co}\left\{z_{k}\right\}_{k=1}^{n}$.

Proof. By defining $w(X)$ and Lemma 3, we can easily obtain the result, so we omit the proof.

By characterizing the relation between weak orthogonal coefficient $w(X)$ and constant $\operatorname{Jin}(X)$, a sufficient condition for Banach space to have normal structure is given.

Theorem 2. If

$$
\operatorname{Jin}(X)<10 w(X)^{2}+4 w(X)+10
$$

then the Banach space $X$ has normal structure. 
Proof. Let $z_{1}$ and $z_{n}$ be as in Lemma 4 , and let

$$
x_{1}=\frac{z_{n}-z_{1}}{\left\|z_{n}-z_{1}\right\|}, x_{2}=\frac{-(w(X)-\varepsilon)\left(z_{n}+z_{1}\right)}{\left\|(w(X)-\varepsilon)\left(z_{n}+z_{1}\right)\right\|}
$$

and

$$
x_{3}=\frac{z_{1}-z_{n}}{\left\|z_{n}-z_{1}\right\|}, x_{4}=\frac{(w(X)-\varepsilon)\left(z_{n}+z_{1}\right)}{\left\|(w(X)-\varepsilon)\left(z_{n}+z_{1}\right)\right\|} .
$$

Then, $x_{1}, x_{2}, x_{3}, x_{4}$ belong to $S_{X}$ and $x_{1}+x_{2}+x_{3}+x_{4}=0$. We set

$$
A=\frac{1}{\left\|z_{n}-z_{1}\right\|}+\frac{w(X)-\varepsilon}{\left\|(w(X)-\varepsilon)\left(z_{n}+z_{1}\right)\right\|}
$$

and

$$
B=\frac{1}{\left\|z_{n}-z_{1}\right\|}-\frac{w(X)-\varepsilon}{\left\|(w(X)-\varepsilon)\left(z_{n}+z_{1}\right)\right\|} .
$$

Then, we obtain

$$
\begin{aligned}
\left\|x_{1}-x_{2}\right\|^{2} & =\left(A\left\|z_{n}-\frac{B}{A} z_{1}\right\|\right)^{2} \\
& =\left(A\left\|z_{n}-\left(\frac{B}{A} z_{1}+\frac{A-B}{A} \cdot 0\right)\right\|\right)^{2} \\
& \geq(A(1-\varepsilon))^{2}
\end{aligned}
$$

and

$$
\begin{aligned}
\left\|x_{1}-x_{4}\right\|^{2} & =\left\|A z_{1}-B z_{n}\right\|^{2} \\
& \geq\left(\left\|A z_{1}\right\|-\left\|B z_{n}\right\|\right)^{2} \\
& =(A-B)^{2} \\
& =\left(\frac{2 w(X)-2 \varepsilon}{\left\|(w(X)-\varepsilon)\left(z_{n}+z_{1}\right)\right\|}\right)^{2} .
\end{aligned}
$$

Similarly, we can deduce

$$
\left\|x_{2}-x_{3}\right\|^{2} \geq\left(\frac{2 w(X)-2 \varepsilon}{\left\|(w(X)-\varepsilon)\left(z_{n}+z_{1}\right)\right\|}\right)^{2},\left\|x_{3}-x_{4}\right\|^{2} \geq(A(1-\varepsilon))^{2} .
$$

Furthermore,

$$
\left\|x_{1}-x_{3}\right\|^{2}=4,\left\|x_{2}-x_{4}\right\|^{2}=4 .
$$

Note that if $\varepsilon \rightarrow 0$, then $A \geq 1+w(X)$. Therefore, from the definition of $\operatorname{Jin}(X)$ and letting $\varepsilon \rightarrow 0$, we have

$$
\operatorname{Jin}(X) \geq 10 w(X)^{2}+4 w(X)+10
$$

In the case of $\operatorname{Jin}(X)<10 w(X)^{2}+4 w(X)+10$, we have $\operatorname{Jin}(X)<24$, and so $X$ is uniformly nonsquare. Therefore, $X$ is reflexive [26], which implies that normal structure equals weak normal structure, as required.

\section{Conclusions}

Based on the characterization of inner product space and the relative background of classical Euclidean geometry, we define a new geometric constant $\operatorname{Jin}(X)$ for a Banach space $X$. It is remarkable that the constant $\operatorname{Jin}(X)$ is symmetric and related to the length of the inscribed quadrilateral side of the unit ball. The introduction of the constant $\operatorname{Jin}(X)$ takes the bridge, attempting to establish a new relationship with the classical constant. In some ways, it provides some ideas for studying the geometric constants of the four variables. 
Author Contributions: Writing-original draft preparation, A.A., Q.L. and Y.L.; writing-review and editing, A.A., Q.L. and Y.L. All authors have read and agreed to the published version of the manuscript.

Funding: This research was funded by the National Natural Science Foundation of China (11971493) and (12071491).

Institutional Review Board Statement: Not applicable.

Informed Consent Statement: Not applicable.

Data Availability Statement: No data were used to support this study.

Acknowledgments: The authors are very grateful to the referees for their many constructive corrections and improvements.

Conflicts of Interest: The authors declare no conflict of interest.

\section{References}

1. Komuro, N.; Saito, K.; Tanaka, R. On the class of Banach spaces with James constant $\sqrt{2}$. Math. Nachrichten 2016, 289, 1005-1020. [CrossRef]

2. Alonso, J.; Martin, P.; Papini, P. Wheeling around von Neumann-Jordan constant in Banach spaces. Stud. Math. 2008, 188, 135-150. [CrossRef]

3. Gao, J. Research on normal structure in a Banach space via some parameters in its dual space. Commun. Korean Math. Soc. 2019, 34, 465-475.

4. Gao, J. On the generalized Pythagorean parameters and the applications in Banach spaces. Discret. Contin. Dyn. Syst. Ser. B 2007, 8, 557-567. [CrossRef]

5. Dinarvand, M. On a generalized geometric constant and sufficient conditions for normal structure in Banach spaces. Acta Math. Sci. 2017, 37, 1209-1220. [CrossRef]

6. Jiménez-Melado, A.; Llorens-Fuster, E.; Saejung, S. The von Neumann-Jordan constant, weak orthogonality and normal structure in Banach spaces. Proc. Am. Math. Soc. 2006, 134, 355-364. [CrossRef]

7. Cho, D.H.; Choi, Y.S. The Bishop-Phelps-Bollobás theorem on bounded closed convex sets. J. Lond. Math. Soc. 2016, 93, 502-518. [CrossRef]

8. Fabiano, C.; Vinícius, M.; Elói, M. A generalized Banach-Stone theorem for $C_{0}(K, X)$ spaces via the modulus of convexity of $X^{*}$. J. Math. Anal. Appl. 2017, 450, 12-20.

9. Tanaka, R. Tingley's problem on symmetric absolute normalized norms on $\mathbb{R}^{2}$. Acta Math. Sin. (Engl. Ser.) 2014, 30, 1324-1340. [CrossRef]

10. Chmieliński, J. Normed spaces equivalent to inner product spaces and stability of functional equations. Aequationes Math. 2014, 87, 147-157 [CrossRef]

11. De la Harpe, P. Classical Banach-Lie Algebras and Banach-Lie Groups of Operators in Hilbert Space; Lecture Notes in Mathematics; Springer: Berlin, Germany; New York, NY, USA, 1972; Volume 285.

12. Zettili, N. Quantum Mechanics: Concepts and Applications; John Wiley \& Sons, Ltd.: Chichester, UK, 2009.

13. Amir, D. Characterizations of Inner Product Spaces, Operator Theory: Advances and Applications; 20 Birkhäuser Verlag: Basel, Switzerland, 1986.

14. Alsina, C.; Sikorska, J.; Tomás, M.S. Norm Derivatives and Characterizations of Inner Product Spaces; World Scientific: Hackensack, NJ, USA, 2010.

15. Moslehian, M.S.; Rassias, J.M. Power and Euler-Lagrange norms. Aust. J. Math. Anal. Appl. 2007, 4, 1-4.

16. Fréchet, M. Sur la definition axiomatique dune classe d'espaces vectoriels distanciés applicables vectoriellement sur l'espace de Hilbert. Ann. Math. 1935, 36, 705-718. [CrossRef]

17. Alonso, J.; Ullán, A. Moduli in normed linear spaces and characterization of inner product spaces. Arch. Math. 1992, 59, 487-495. [CrossRef]

18. Nikodem, K.; Páles, Z.S. Characterizations of inner product spaces by strongly convex functions. Banach J. Math. Anal. 2011, 5, 83-87. [CrossRef]

19. Day, M.M. Some characterizations of inner-product spaces. Trans. Am. Math. Soc. 1947, 62, 320-337. [CrossRef]

20. Jordan, P.; von Neumann, J. On inner products in linear metric spaces. Ann. Math. 1935, 36, 719-723. [CrossRef]

21. Clarkson, J.A. The von Neumann-Jordan constant for the Lebesgue space. Ann. Math. 1937, 38, 114-115. [CrossRef]

22. Gao, J.; Lau, K.S. On the geometry of spheres in normed linear spaces. J. Aust. Math. Soc. Ser. A 1990, 48, 101-112. [CrossRef]

23. Kato, M.; Takahashi, Y. On the von Neumann-Jordan constant for Banach spaces. Proc. Am. Math. Soc. 1997, 125, 1055-1062. [CrossRef]

24. Kato, M.; Maligranda, L.; Takahashi, Y. On James Jordan-Von Neumann constants and the normal structure coefficient of Banach spaces. Stud. Math. 2001, 144, 275-295. [CrossRef] 
25. Takahashi, Y. Some geometric constants of Banach spaces-A unified approach. In Banach and Function Spaces II; Kato, M., Maligranda, L., Eds.; Yokohama Publishers: Yokohama, Japan, 2007; pp. 191-220.

26. James, R.C. Uniformly non-square Banach spaces. Ann. Math. 1964, 80, 542-550. [CrossRef]

27. Kolwicz, P.; Panfil, A. Non-square Lorentz spaces $\Gamma_{p, \omega}$, Indag. Math. (N.S.) 2013, 24, 254-263. [CrossRef]

28. Baronti, M.; Casini, E.; Papini, P.L. Triangles Inscribed in a Semicircle, in Minkowski Planes, and in Normed Spaces. J. Math. Anal. Appl. 2000, 252, 124-146. [CrossRef]

29. Alonso, J. Llorens-Fuster, E. Geometric mean and triangles inscribed in a semicircle in Banach spaces. J. Math. Anal. Appl. 2008, 340, 1271-1283. [CrossRef]

30. Fu, Y.; Liu, Q.; Li, Y. New Geometric Constants in Banach Spaces Related to the Inscribed Equilateral Triangles of Unit Balls. Symmetry 2021, 13, 951. [CrossRef]

31. Mizuguchi, H. Some geometric constants and the extreme points of the unit ball of Banach spaces. Rev. Roum. Math. Pures Appl. 2015, 60, 59-70.

32. Olaru, I.M.; Secelean, N.A. A New Approach of Some Contractive Mappings on Metric Spaces. Mathematics 2021, 9 , 1433. [CrossRef]

33. Ersin, G.; Diana, D.; Zoran, D.M.; Dženis, P.; Hassen, A. On Some Recent Results Concerning F-Suzuki-Contractions in b-Metric Spaces. Mathematics 2020, 8, 940 .

34. Jäntschi, L.; Bálint, D.; Bolboaca, S. Multiple Linear Regressions by Maximizing the Likelihood under Assumption of Generalized Gauss-Laplace Distribution of the Error. Comput. Math. Methods Med. 2016, 2016, 1-8. [CrossRef] [PubMed]

35. García-Falset, J.; Llorens-Fuster, E.; Mazcunán-Navarro, E.M. Uniformly nonsquare Banach spaces have the fixed point property for nonexpansive mappings. J. Funct. Anal. 2006, 233, 494-514. [CrossRef]

36. Brodskii, M.; Milman, D. On the center of a convex Set. Dokl. Akad. Nauk SSSR (N.S.) 1948, 59, 837-840.

37. Prus, S. Geometrical background of metric fixed point theory. In Handbook of Metric Fixed Point Theory; Kirk, W.A., Sims, B., Eds.; Kluwer Acad. Publ.: Dordrecht, The Netherlands, 2001; pp. 93-132.

38. Sims, B. Orthogonality and fixed points of nonexpansive maps. Proc. Cent. Austral. Nat. Univ. 1988, 20, 179-186.

39. Sims, B. A class of spaces with weak normal structure. Bull. Austral. Math. Soc. 1994, 50, 523-528. [CrossRef]

40. Dulst, V. Some more Banach spaces with normal structure. J. Math. Anal. Appl. 1984, 104, 285-289. [CrossRef] 